\title{
A FORMAL MELLIN TRANSFORM IN THE ARITHMETIC OF FUNCTION FIELDS
}

\author{
DAVID GOSS
}

\begin{abstract}
The Mellin transform is a fundamental tool of classical arithmetic. We would also like such a tool in the arithmetic of function fields based on Drinfeld modules, although a construction has not yet been found. One formal approach to finding Mellin transforms in classical theory is through $p$-adic measures. It turns out that this approach also works for function fields. Thus this paper is devoted to exploring what can be learned this way. We will establish some very enticing connections with gamma functions and the KummerVandiver conjecture for function fields.
\end{abstract}

\section{INTRODUCTION}

One of the most important tools of classical number theory is the Mellin transform which takes Dirichlet series and turns them into algebraic objects (i.e., modular forms or formal power series). Mellin transforms are essential in proofs of functional equations, for reciprocity laws, etc.

If one did not know the construction of the Mellin transform through complex analysis, one would still have some knowledge of it p-adically. Indeed, the transform of $n^{-s}$ is formally $T^{n}$, whether one uses complex analysis $o r$ nonarchimedean analysis (where it is precisely the Dirac measure, see $\S 2$ ). Of course, the classical theory does exist but as yet its analog in the theory of function fields does not. Recently, however, there has been progress in the analog of $p$-adic measures for function fields. Thus, the purpose of this short paper is to investigate what this tells us about the potential shape a "Mellin transform" should take in the theory. We will present results that form the basis for future inquiry in a number of areas.

Let $\mathbf{A}=\mathbf{F}_{r}[T], \mathbf{k}=\mathbf{F}_{r}(T)$, and $\mathbf{K}=\mathbf{k}_{\infty}=\mathbf{F}_{r}\left(\left(\frac{1}{T}\right)\right)$. Let $v \in \operatorname{Spec}(\mathbf{A})$. After recalling the classical situation in $\S 2$, we review some results from [6]; the fundamental result obtained there tells us that $v$-adic measures on $\mathbf{A}_{v}$ correspond to divided power series where $p$-adically one obtains formal power series. We then examine the divided power series coming from Dirac measures and these will turn out to be very well behaved. For instance, they will give us a new form

Received by the editors March 10, 1989 and, in revised form, February 27, 1990.

1980 Mathematics Subject Classification (1985 Revision). Primary 12A90.

Key words and phrases. Mellin transforms, nonarchimedean measures, divided power series, hyper-derivatives, zeta functions, gamma functions, magic numbers.

Partially supported by NSF Grant DMS-8521678. 
of exponentiation for function fields. It is the theory of Dirac measures that allows us to define the "formal Mellin transforms" for function fields (as well as number fields).

Divided power series have a dual identity as "hyper-derivatives," and this will allow us to obtain an action of $\mathbf{K}$ on certain measure algebras. This action then gives rise, in a natural fashion, to an integral formula for the author's gamma function (Theorem 3.24). The classical complex theory leads us to expect that this formula fits into a much larger edifice whose exact structure is not yet clear.

Lastly we present the computation of the formal Mellin transform in the simplest possible situation. These results, which were implied by various calculations, have recently been established by Dinesh Thakur [10]. They are remarkable for their simplicity and for their completely unexpected connection with magic numbers (magic numbers are certain integers which arise in the Kummer-Vandiver conjecture for function fields). We also present a result that sets the stage for further calculations in the area of analogs of Dedekind zeta functions. It may turn out that the simplicity of Thakur's results indicates a nonobvious (at this point) connection with characteristic 0 .

In a forthcoming work, [6], we will use the ideas introduced here to develop a Fourier transform and an associated Poisson summation formula for function fields.

During the writing of this paper, the author had the pleasure of conversations with D. Hayes, N. Katz, M. Rosen, W. Sinnott, and D. Thakur. We also thank the referee for useful expository suggestions.

\section{Classical theory}

Let $\Gamma(s)=\int_{0}^{\infty} t^{s-1} e^{-t} d t$ be Euler's gamma function, and let $\zeta(s)=\sum_{n=1}^{\infty} n^{-s}$ be Riemann's zeta function. As is very well known, for $\Re(s)>1$

$$
n^{-s} \Gamma(s)=\int_{0}^{\infty} x^{s} e^{-n x} \frac{d x}{x}
$$

thus

$$
\zeta(s) \Gamma(s)=\int_{0}^{\infty} x^{s}\left(\sum_{n=1}^{\infty} e^{-n x}\right) \frac{d x}{x}=\int_{0}^{\infty} x^{s}\left(\sum_{n=1}^{\infty}\left(e^{-x}\right)^{n}\right) \frac{d x}{x} .
$$

As $T+T^{2}+\cdots=\frac{T}{1-T}$; we have, upon substituting $e^{-x}$ for $T$,

$$
\zeta(s) \Gamma(s)=\int_{0}^{\infty} x^{s} \frac{e^{-x}}{1-e^{-x}} \frac{d x}{x} .
$$

This formula, and the related one involving $\Gamma(s), \zeta(s)$, and the theta function, $\vartheta(s)=\frac{1}{2} \sum_{n=-\infty}^{\infty} e^{\pi i n^{2} s}$, lie at the heart of applications of analysis to $\zeta(s)$ (and, for that matter, to all $L$-series).

The theta function, $\vartheta(s)$, may be recovered "algebraically" as a modular form of weight $\frac{1}{2}$ for a certain subgroup of $\mathrm{SL}_{2}(Z)$ of finite index. We now 
recall how to recover the rational function $r(T)=\frac{T}{1-T}$ "algebraically" through the use of nonarchimedean measures (for proofs, see [9]).

Let $p$ be a prime of $\mathbf{Z}$ with associated completion $\mathbf{Z}_{p}$.

Definition 2.1. (a) We put $\left(\begin{array}{l}x \\ 0\end{array}\right)=1$.

(b) Let $k \geq 1$. We put

$$
\left(\begin{array}{l}
x \\
k
\end{array}\right)=\frac{x(x-1) \cdots(x-k+1)}{k !} .
$$

As $\left(\begin{array}{l}x \\ k\end{array}\right): \mathbf{Z} \rightarrow \mathbf{Z}$, continuity implies that $\left(\begin{array}{l}x \\ k\end{array}\right): \mathbf{Z}_{p} \rightarrow \mathbf{Z}_{p}$ for all primes $p$. We then have the following classical result due to K. Mahler:

Theorem 2.2. Let $f(x): \mathbf{Z}_{p} \rightarrow \mathbf{Q}_{p}$ be a continuous function. Then $f(x)$ can be written uniquely as

$$
f(x)=\sum_{k \geq 0} a_{k}\left(\begin{array}{l}
x \\
k
\end{array}\right),
$$

with $\left\{a_{k}\right\} \subset \mathbf{Q}_{p}$, and $a_{k} \rightarrow 0$ as $k \rightarrow \infty$. Conversely, any sequence $\left\{a_{k}\right\}$, as above, will uniquely determine a continuous function.

Definition 2.3. A $\mathbf{Z}_{p}$-valued measure $\mu$ on $\mathbf{Z}_{p}$ is a finitely additive $\mathbf{Z}_{p}$-valued function on the compact open subsets of $\mathbf{Z}_{p}$.

Remark 2.4. (a) The above definition is equivalent to the definition given in $\S 4$ of [9] in terms of "distributions." The reader may see this as follows: A $\mathbf{Z}_{p}$-valued distribution on $\mathbf{Z}_{p}$ is a sequence of functions

$$
\nu_{j}: \mathbf{Z} /\left(p^{j}\right) \rightarrow \mathbf{Z}_{p}, \quad \text { for } j>0,
$$

which is compatible in the sense that, if we denote by $\pi_{j}$ the projection of $\mathbf{Z} /\left(p^{j}\right)$ onto $\mathbf{Z} /\left(p^{j-1}\right)$, then

$$
\nu_{j}(x)=\sum_{\pi_{j+1} y=x} \nu_{j+1}(y) .
$$

In this approach, " $\mathbf{Z}_{p}$-valued distributions on $\mathbf{Z}_{p}$ " and " $\mathbf{Z}_{p}$-valued measures on $\mathbf{Z}_{p}$ " have the same meaning.

Now let $\mu$ be a measure in the sense of Definition 2.3. To $\mu$ we associate the sequence of functions $\mu_{j}: \mathbf{Z} /\left(p^{j}\right) \rightarrow \mathbf{Z}_{p}$ defined by

$$
\mu_{j}\left(a+\left(p^{j}\right)\right)=\mu\left(a+\left(p^{j}\right)\right) .
$$

The finite additivity of $\mu$ precisely implies that $\left\{\mu_{j}\right\}$ is a distribution.

Conversely, any compact open $U \subset \mathbf{Z}_{p}$ is a finite union of cosets $\bmod \left(p^{j}\right)$ for some $j$. Thus equation (2.5) also tells us how to attach a measure to any distribution. This establishes the equivalence.

(b) Definition 2.3 generalizes to the notion of $\mathbf{Q}_{p}$-valued measures on $\mathbf{Z}_{p}$ as follows: One no longer assumes that, for $U$ a compact open $\subseteq \mathbf{Z}_{p}, \mu(U) \in \mathbf{Z}_{p}$, but rather that

$$
\{\mu(U)\}_{U \subseteq \mathbf{Z}_{p}}
$$


is bounded. This is equivalent to assuming that there exist some $\lambda \in \mathbf{Z}_{p}$ such that $\lambda \mu$ is a $\mathbf{Z}_{p}$-valued measure on $\mathbf{Z}_{p}$.

We let $M\left(\mathbf{Z}_{p}\right)$ denote the module of $\mathbf{Z}_{p}$-valued measures on $\mathbf{Z}_{p}$, and we let $B M\left(\mathbf{Z}_{p}\right)$ be the vector space of $\mathbf{Q}_{p}$-valued measures. Thus, by definition,

$$
B M\left(\mathbf{Z}_{p}\right)=\mathbf{Q}_{p} \otimes M\left(\mathbf{Z}_{p}\right) .
$$

Let $f(x)$ be a continuous $\mathbf{Q}_{p}$-valued function on $\mathbf{Z}_{p}$, and let $\mu \in B M\left(\mathbf{Z}_{p}\right)$. By using step-function approximations, we can form the Riemann integral

$$
\int_{\mathbf{z}_{p}} f(x) d \mu(x)
$$

with respect to $\mu$. As the continuous functions from $\mathbf{Z}_{p}$ to $\mathbf{Q}_{p}$ can be uniformly approximated by step-functions, one sees directly that $B M\left(\mathbf{Z}_{p}\right)$ is dual to the space of continuous functions. Thus a $p$-adic measure $\mu$ is uniquely determined by the sequence of integrals

$$
\left\{b_{k}=\int_{\mathbf{z}_{p}}\left(\begin{array}{l}
x \\
k
\end{array}\right) d \mu(x)\right\} \subset \mathbf{Q}_{p},
$$

where $n$ runs over nonnegative integers. Note that, by definition, the set $\left\{b_{k}\right\}$ is bounded. Conversely, any such bounded sequence uniquely determines a measure.

Definition 2.6. Let $\mu_{1}, \mu_{2}$ be any two $\mathbf{Q}_{p}$-valued measures on $\mathbf{Z}_{p}$. We define their convolution $\mu_{1} * \mu_{2}$ to be the measure defined by

$$
\int_{\mathbf{Z}_{p}} f(x) d\left(\mu_{1} * \mu_{2}\right)(x)=\int_{\mathbf{Z}_{p}} \int_{\mathbf{Z}_{p}} f(x+t) d \mu_{1}(x) d \mu_{2}(t),
$$

for any continuous function $f(x)$.

Now let $\mu \in B M\left(\mathbf{Z}_{p}\right)$. We associate to $\mu=\left\{b_{k}\right\}$ the power series

$$
P_{\mu}(X)=\sum_{k \geq 0} b_{k} X^{k}
$$

It then follows directly from elementary properties of $\left(\begin{array}{l}x \\ k\end{array}\right)$ that $M\left(\mathbf{Z}_{p}\right)$, with convolution, is canonically isomorphic to the ring $\Lambda=\Lambda_{p}=\mathbf{Z}_{p}[[X]]$. Moreover, $B M\left(\mathbf{Z}_{p}\right)$ then becomes isomorphic to

$$
\mathbf{Q}_{p} \otimes \Lambda_{p}=\mathbf{Q}_{p} \otimes \mathbf{Z}_{p}[[X]]
$$

i.e., the algebra of power series with bounded denominators.

Let $\mu \in B M\left(\mathbf{Z}_{p}\right)$ and let $P_{\mu}(X)$ be as above. Let $z \in p \mathbf{Z}_{p}$. Then we have

Lemma 2.7.

$$
\int_{\mathbf{Z}_{p}}(1+z)^{x} d \mu(x)=P_{\mu}(z) .
$$

Proof. Expand $(1+z)^{x}$ by the binomial theorem and use the definition of $P_{\mu}(X)$. 
Remark 2.8. (a) Lemma 2.7 is equivalent to the formal identity on power series

$$
\int_{\mathbf{Z}_{p}}(1+X)^{x} d \mu(x)=P_{\mu}(X) .
$$

(b) Let $\mathscr{M} \subset \Lambda_{p}$ be the ideal generated by $\{p, X\}$. One then gives $\Lambda_{p}$ the $\mathscr{M}$-adic topology with which it is complete. Similarly, one topologizes $\mathbf{Q}_{p} \otimes \Lambda_{p}$. With these topologies, it is easy to see that the word "formal" can be removed from (a).

The reader should note that the $\mathscr{M}$-adic topology on $\Lambda_{p}$ is precisely the topology it inherits from the distribution definition of measures; i.e., the identification of $\Lambda_{p}$ with the "Iwasawa algebra" of $\mathbf{Z}_{p}$ (see [9]).

Let $\alpha \in \mathbf{Z}_{p}$. Associated to $\alpha$ we have the Dirac measure $\delta_{\alpha}$ defined by

$$
\int_{\mathbf{Z}_{p}} f(x) d \delta_{\alpha}(x)=f(\alpha),
$$

for any $f$. Thus, for compact open subsets $U \subseteq \mathbf{Z}_{p}$ we have

$$
\delta_{\alpha}(U)= \begin{cases}1 & \text { if } \alpha \in U \\ 0 & \text { otherwise }\end{cases}
$$

The next result is well known:

Lemma 2.9. $P_{\delta_{r}}(X)=(1+X)^{\alpha}$.

Proof. By definition

$$
\int\left(\begin{array}{l}
x \\
k
\end{array}\right) d_{\delta_{\alpha}}(x)=\left(\begin{array}{l}
\alpha \\
k
\end{array}\right) .
$$

Thus the result follows immediately.

We set $P_{\alpha}(X)=P_{\delta_{\alpha}}(X)$ and $T=1+X$. Then we now have the following obvious result:

Proposition 2.10. We have formally

$$
r(T)=\frac{T}{1-T}=\frac{1+X}{-X}=\sum_{n=1}^{\infty} P_{n}(X) .
$$

Let $\Lambda_{1}=\Lambda\left[\frac{1}{X}\right]$. Then clearly $r(T)=\frac{T}{1-T}=\frac{1+X}{-X}$ exists as an element of $\Lambda_{1}$. (The reader should note, however, that the sum does not converge as elements of $\Lambda_{1}$. Moreover, though we have worked $p$-adically, the answer $r(T)$ clearly is invariant of $p$.$) The point of these calculations is that the rational function$ $r(T) \in \Lambda_{1}$ appears as the "formal ( $p$-adic) Mellin transform of $\zeta(s)$." Of course, in order to analytically continue $\zeta(s)$ to the whole complex plane, one needs to alter the function $r(T)$ slightly. However, this altered function can also be recovered $p$-adically as an element of $\Lambda$ (not just $\Lambda_{1}$, see [8]). Once 
this is accomplished, one then obtains (slightly modified) special values of $\zeta(s)$ at negative integers as the moments of this measure.

\section{THE THEORY FOR FUNCTION FIELDS}

3.1. Review. In this section we will recall, without proofs, some results due to L. Carlitz, C. Wagner, and the author. For more information, the reader can consult [6].

We set $\mathbf{A}=\mathbf{F}_{r}[T], r=p^{m}, p$ a rational prime, $\mathbf{k}=\mathbf{F}_{r}(T)$, and $\mathbf{K}=\mathbf{k}_{\infty}=$ $\mathbf{F}_{r}\left(\left(\frac{1}{T}\right)\right)=$ the completion of $\mathbf{k}$ with respect to the prime $\infty$ of $\mathbf{k}$. Let $v=(f)$ be a prime of $\mathbf{A}$. We denote by $\mathbf{A}_{v}$ the completion of $\mathbf{A}$ with respect to $v$ and by $\mathbf{k}_{v}$ the completion of $\mathbf{k}$. Finally, we abuse notation and set $\mathbf{A}_{\infty}$ to be the ring of integers in $\mathbf{K}$.

Definition 3.1. (a) For $j>0$ we set $[j]=T^{r^{j}}-T$.

(b) We set $D_{0}=L_{0}=1$. For $j \geq 1$, we set

$$
D_{j}=[j][j-1]^{r} \cdots[1]^{r^{j-1}}, \text { and } L_{j}=[j][j-1] \cdots[1] \text {. }
$$

(c) We set $e_{0}(x)=x$ and for $j \geq 1$, we set

$$
e_{j}(x)=\prod_{\substack{\alpha \in \mathrm{A} \\ \operatorname{deg}(\alpha)<j}}(x-\alpha) .
$$

(d) Let $\sum_{t=0}^{\nu} \alpha_{t} r^{t}$ be the $r$-adic expansion of $i$. We set

$$
\Pi(i)=\prod_{t=0}^{\nu} D_{t}^{\alpha_{t}}, \quad \text { and } \quad G_{i}(x)=\prod_{t=0}^{\nu} e_{t}(x)^{\alpha_{t}} .
$$

(e) Let $i$ be as above. We set

$$
g_{i}(x)=\prod g_{\alpha_{r} r^{r}}(x),
$$

where

$$
g_{\alpha_{t} r^{r}}(x)= \begin{cases}e_{t}^{\alpha_{t}}(x) & \text { if } \alpha_{t}<r-1, \\ e_{t}^{r-1}(x)-D_{i}^{r-1} & \text { if } \alpha_{t}=r-1 .\end{cases}
$$

The reader should note that we have changed notation from our earlier papers $[2,5,6]$; we now use $\Pi(i)$ where before we used $\Gamma_{i}$. The change is to avoid possible confusion arising from the fact that the interpolated $\Gamma$-functions of $[5$, 6] are defined through the use of a shift $s \mapsto s-1$.

One sees directly that the $e_{j}(x)$ are $\mathbf{F}_{r}$-linear polynomials. The next statement gives an explicit formula for them:

Proposition 3.2. Let $i, j$ be nonnegative integers. If $i>j$, put $\left[\begin{array}{l}j \\ i\end{array}\right]=0$. If $i \leq j$, put

$$
\left[\begin{array}{c}
j \\
i
\end{array}\right]=\frac{D_{j}}{D_{i} L_{j-i}^{r^{i}}}
$$


Then

$$
e_{j}(x)=\sum_{t=0}^{j}(-1)^{j-t}\left[\begin{array}{l}
j \\
t
\end{array}\right] x^{r^{t}} .
$$

The functions $G_{i}(x)$ are called the Carlitz functions. Carlitz has shown that if $\alpha \in \mathbf{A}$ then $\frac{G_{k}(\alpha)}{\Pi(k)}$ does also. Thus, $\frac{G_{k}(x)}{\Pi(k)}$ is a continuous function from $\mathbf{A}_{v}$ to itself-exactly similar to what happens for $\left(\begin{array}{l}x \\ k\end{array}\right) p$-adically.

We then have the following result due to $C$. Wagner. It is a function field analog of Mahler's result Theorem 2.2.

Theorem 3.3. Let $f(x): \mathbf{A}_{v} \rightarrow \mathbf{k}_{v}$ be a continuous function. Then

$$
f(x)=\sum_{k \geq 0} a_{k} \frac{G_{k}(x)}{\Pi(k)},
$$

with $\left\{a_{k}\right\} \subset \mathbf{k}_{v}$, and $a_{k} \rightarrow 0$ as $k \rightarrow \infty$. Conversely, any such sequence $\left\{a_{k}\right\}$ determines a continuous function as above.

It is easy to see that the above result extends in the obvious fashion to continuous functions which have their values in finite extensions of $\mathbf{k}_{v}$.

There is also a remarkable "integral" formula for the coefficients $\left\{a_{k}\right\}$, due to Carlitz, which we now recall:

Proposition 3.4. Let $k \geq 0$ and let $r^{m}>k$. Then

$$
a_{k}=(-1)^{m} \sum_{\operatorname{deg}(\alpha)<m} \frac{g_{r^{m}-1-k}(\alpha)}{\Pi\left(r^{m}-1-k\right)} f(\alpha) .
$$

Moreover, Carlitz established the following "Leibnizian" addition law for the $G_{j}(x)$ :

\section{Proposition 3.5.}

$$
G_{j}(x+u)=\sum_{e+f=j}\left(\begin{array}{l}
j \\
e
\end{array}\right) G_{e}(x) G_{f}(u) .
$$

We leave the obvious generalization of Definition 2.3 to $\mathbf{A}_{v}$-valued measures on $\mathbf{A}_{v}$ (or $\mathbf{Z}_{p}$ ), as well as their convolutions, etc., to the reader. We let $M_{v}\left(\mathbf{A}_{v}\right)$ (resp. $M_{v}\left(\mathbf{Z}_{p}\right)$ ) be the convolution ring of $\mathbf{A}_{v}$-valued measures on $\mathbf{A}_{v}$ (resp. $\left.\mathbf{Z}_{p}\right)$. We also let $B M_{v}\left(\mathbf{A}_{v}\right)$ (resp. $B M_{v}\left(\mathbf{Z}_{p}\right)$ ) be the convolution ring of $\mathbf{k}_{v}$ valued measures on $\mathbf{A}_{v}$ (resp. $\mathbf{Z}_{p}$ ); thus $B M_{v}\left(\mathbf{A}_{v}\right)=\mathbf{k}_{v} \otimes M_{v}\left(\mathbf{A}_{v}\right)$, etc. Finally, with the obvious definitions, we let $M_{\infty}\left(\mathbf{A}_{\infty}\right)$ (resp. $\left.M_{\infty}\left(\mathbf{Z}_{p}\right)\right)$ denote the ring of $\mathbf{A}_{\infty}$-valued measures on $\mathbf{A}_{\infty}$ (resp. $\mathbf{Z}_{p}$ ) and so on.

Let $\mu \in B M_{v}\left(\mathbf{A}_{v}\right)$. To $\mu$ we associate the bounded sequence

$$
\left\{b_{k}=\int_{\mathbf{A}_{v}} \frac{G_{k}(x)}{\Pi(k)} d \mu(x)\right\} \subset \mathbf{k}_{v} .
$$

By the result of Wagner, Theorem 3.3, this sequence uniquely determines $\mu$. Moreover, classically, any such bounded sequence will uniquely determine a 
measure. As $G_{k}(x)$ is a polynomial in $x$, it is clear that $\mu$ is uniquely determined by its moments

$$
\int_{\mathbf{A}_{v}} x^{n} d \mu(x)
$$

as $n$ runs over the nonnegative integers.

To $\mu=\left\{b_{k}\right\}$ we associate the divided power series

$$
P_{\mu}(Z)=\sum_{k \geq 0} b_{k} \frac{Z^{k}}{k !} .
$$

The reader should recall that, although " $\frac{Z^{k}}{k !}$ " does not make literal sense (since $k$ ! will usually be 0 in $\mathbf{A}$ ), the multiplication of two such symbols does make sense! Our Leibnizian addition rule, Proposition 3.5 given above, then tells us that $M_{v}\left(\mathbf{A}_{v}\right)$ is canonically isomorphic to the ring $\Delta=\Delta_{v}$ of formal divided power series over $\mathbf{A}_{v}$. Moreover, $B M_{v}\left(\mathbf{A}_{v}\right)$ is then canonically isomorphic to the ring

$$
\mathbf{k}_{v} \otimes M_{v}\left(\mathbf{A}_{v}\right)
$$

i.e., the ring of formal divided power series with bounded denominators.

It is easily checked that the classical result of Mahler, Theorem 2.2, can be altered to show that $M_{v}\left(\mathbf{Z}_{p}\right)$ is isomorphic to the formal power series ring $\mathbf{A}_{v}[[X]]$ over $\mathbf{A}_{v}$ and that $M_{\infty}\left(\mathbf{Z}_{p}\right)$ is isomorphic to $\mathbf{A}_{\infty}[[X]]$ (see [16]), etc. We will call these rings $\Lambda_{v}$ and $\Lambda_{\infty}$, respectively. It follows that the Dirac measure at $\alpha$ still corresponds to the power series $(1+X)^{\alpha}$. Moreover, once we have chosen a uniformizer $\pi=\frac{1}{T}+c \frac{1}{T^{2}}+\cdots$ at $\infty$, the result of Wagner is trivially modified to show that $M_{\infty}\left(\mathbf{A}_{\infty}\right)$ is also a divided power series algebra.

Let $\alpha \in \mathbf{A}_{v}$ and let $\delta_{\alpha}$ be the Dirac measure supported at $\alpha$ with the obvious definition. Then we have

Proposition 3.6.

$$
P_{\delta_{r}}(Z)=\sum_{k \geq 0} \frac{G_{k}(\alpha)}{\Pi(k)} \frac{Z^{k}}{k !} .
$$

Proof. Clearly,

$$
\int_{\mathbf{A}_{v}} \frac{G_{k}(x)}{\Pi(k)} d \delta_{\alpha}(x)=\frac{G_{k}(\alpha)}{\Pi(k)} .
$$

Thus the result is obvious.

As before, we set $P_{\alpha}(Z)=P_{\delta_{n}}(Z)$.

Proposition 3.7. Let $\alpha, \beta$ be in $\mathbf{A}_{v}$. Then

$$
P_{\alpha+\beta}(Z)=P_{\alpha}(Z) P_{\beta}(Z) \text {. }
$$

Proof. This follows directly from the definition of convolution.

In the case of $\mathbf{Q}_{p}$-valued measures on $\mathbf{Z}_{p}$ the corresponding result on Dirac measures just gives the well-known property of exponentiation!

Next, let $a \in \mathbf{A}$. Then we have 
Proposition 3.8. $P_{a}(Z)$ is a "divided polynomial;" i.e., contains only finitely many terms $\frac{Z^{k}}{k !}$. Moreover, only elements of $\mathbf{A}$ have this property.

Proof. Clearly, $G_{k}(a)=0$ for all sufficiently large $k$. On the other hand, if $\alpha \notin \mathbf{A}$ then $G_{k}(\alpha)$ is never 0 .

The reader should again compare with the classical $p$-adic situation where it is obvious that $P_{\alpha}(X) \in \mathbf{Z}_{p}[X]$ implies that $\alpha$ is a nonnegative integer.

We also have the following analog of Lemma 2.7 as interpreted in Remark 2.8. I thank W. Sinnott for pointing it out to me.

Lemma 3.9. Let $\mu$ be a $\mathbf{k}_{v}$-valued measure on $\mathbf{A}_{v}$. Let $P_{\mu}(Z)$ be the divided power series associated to $\mu$. Then we have formally

$$
\int_{\mathbf{A}_{v}} P_{x}(Z) d \mu(x)=P_{\mu}(Z) .
$$

Remark 3.10. As in Remark 2.8, one can give $\Delta_{v}$ its canonical linear topology as the Iwasawa algebra of $\mathbf{A}_{v}$. Using this, one can remove the word "formally" from Lemma 3.9. However, unlike, the classical situation of $\mathbf{Z}_{p}$-valued measures on $\mathbf{Z}_{p}$, this topology is not an $\mathscr{M}$-adic topology for some ideal $\mathscr{M}$. As such, and as Lemma 3.9 will not be needed later on, we will leave the (messy!) details to the reader.

Finally, let $F_{p}$ denote the $p$ th-power map: $F_{p}(x)=x^{p}$. There is an interesting action of this mapping on our measure algebras. Indeed, let $Y$ be one of the spaces $\left\{\mathbf{Z}_{p}, \mathbf{A}_{v}\right\}$, etc., that we have defined characteristic $p$-valued measures on and let $\mu$ be one such measure. We let $\mathscr{F}$ be the complete field containing the values of $\mu$; thus $\mathscr{F}$ will be one of the completions of $\mathbf{k}$.

Definition 3.11. We define $\mu^{F}$ by

$$
\mu^{F}(U)=F_{p}(\mu(U)),
$$

for any $U \subset Y$ which is compact and open.

It is obvious that $(\mu+\nu)^{F}=\mu^{F}+\nu^{F}$. Moreover, it is clear that $\mu^{F}=\mu$ if $\mu$ is a Dirac measure.

Lemma 3.12. Let $Y, \mu$, and $\mathscr{F}$ be as above. Let $f(x): X \rightarrow \mathscr{F}$ be a continuous function. Then

$$
F_{p}\left(\int_{Y} f(x) d \mu(x)\right)=\int_{Y} f(x)^{p} d \mu^{F}(x) .
$$

Proof. The integral is defined as the limit of Riemann sums. The result follows easily from this.

Now let $\mu, \nu$ be two measures on $Y$, and recall that we set $\mu * \nu$ to be their convolution.

Proposition 3.13. We have

$$
(\mu * \nu)^{F}=\mu^{F} * \nu^{F}
$$


Thus the mapping $F_{p}$ gives rise to a homomorphism of measure algebras.

Proof. Let $U \subset Y$ be any compact open subset and let $\chi_{U}(x)$ be the characteristic function of $U$. Then we need only check that

$$
\int_{Y} \chi_{U}(x) d(\mu * \nu)^{F}(x)=\int_{Y} \chi_{U}(x) d\left(\mu^{F} * \nu^{F}\right)(x),
$$

for all such $U$. But this follows immediately from Lemma 3.12. have:

We can compute explicitly the action of $F_{p}$ in the case $Y=\mathbf{Z}_{p}$. In fact we

Proposition 3.14. Let $\mathscr{F}$ be as above, and let $\mu$ be an $\mathscr{F}$-valued measure on $\mathbf{Z}_{p}$. Let $P_{\mu}(X)=\sum b_{k} X^{k}$. Then

$$
P_{\mu^{F}}(X)=\sum b_{k}^{p} X^{k}
$$

Proof. By definition

$$
b_{k}=\int_{\mathbf{Z}_{p}}\left(\begin{array}{l}
x \\
k
\end{array}\right) d \mu(x) .
$$

Note that $\left(\begin{array}{l}x \\ k\end{array}\right)$ takes values in $\mathbf{Z} /(p)$. Thus the result follows immediately from Lemma 3.12.

In principle we can use Wagner's result to compute the action of $F_{p}$ on a measure $\mu$ on $\mathbf{A}_{v}$ or $\mathbf{A}_{\infty}$. Indeed, we have seen that we need only compute the moments of $\mu^{F}$. Let $n$ be a nonnegative integer. Clearly

$$
F_{p}\left(\int x^{n / p} d \mu(x)\right)=\int x^{n} d \mu^{F}(x) .
$$

Now Wagner's result, and Carlitz's integral formula, tell us how to expand the continuous function $x^{n / p}$ in terms of the functions $\frac{G_{j}(x)}{\Pi(j)}$. Thus, we can, in theory, read off the moments of $\mu^{F}$. However, this looks like it may be difficult to work out in practice.

The reason that the action of $F_{p}$ on the algebra of measures on $\mathbf{Z}_{p}$ is so simple is the following: Let $\kappa$ be any continuous homomorphism of $\mathbf{A}_{v}$ to a $p$-adically complete ring. Then the arguments given above for $F_{p}$ are easily modified to establish that $\kappa$ gives rise to an algebra homomorphism $\mu \rightarrow \mu^{\kappa}$ of measures on $\mathbf{Z}_{p}$. Furthermore, Mahler's description of continuous functions works well over any $p$-adically complete ring. Thus the induced action of $\kappa$ on power series just corresponds to its action on constants.

Now let $R$ be any $v$-adically complete extension of $\mathbf{A}_{v}$. The argument given above for $\kappa$ and measures on $\mathbf{Z}_{p}$ works for any A-algebra homomorphism of $R$ to any $v$-adically complete ring and $R$-valued measures on $\mathbf{A}_{v}$. The problem is that $F_{p}$ is, of course, not A-linear.

3.2. The connection with hyper-derivatives. We shall now see how the interpretation of $v$-adic measures as divided power series provides us with a remarkable connection to differential operators. 
Definition 3.15. Let $R$ be a ring, and let $D=\frac{d}{d X}$. We set $R\{\{D\}\}$ to be the $R$-algebra of formal differential operators

$$
\sum_{k \geq 0} a_{k} \frac{D^{k}}{k !}=\sum_{k \geq 0} a_{k} \frac{\left(\frac{d}{d X}\right)^{k}}{k !} .
$$

We call the ring $R\{\{D\}\}$ the algebra of hyper-derivatives over $R$.

As with the definition of divided power series, the elements “ $\frac{D^{k}}{k !}$ " do not make literal sense. However, their action on polynomials (or power series) over $R$ is well defined in the obvious manner.

Hyper-derivatives are of great utility because they are the "best" one can expect from the calculus in finite characteristic. Indeed, in characteristic $p$, $\left(\frac{d}{d X}\right)^{p}$ is identically 0 , and the hyper-derivatives remedy, as far as possible, this sad state of affairs. It is important to note, however, that in characteristic $p$ the algebra of hyper-derivatives is most definitely not generated by $D$.

Our next result follows directly.

Proposition 3.16. Let $R=\mathbf{A}_{v}$. Then there is an isomorphism $\Delta \rightarrow \mathbf{A}_{v}\{\{D\}\}$ given by

$$
P(Z)=\sum a_{k} \frac{Z^{k}}{k !} \mapsto P(D)=\sum a_{k} \frac{D^{k}}{k !} .
$$

Note that if $P(Z)$ is a divided polynomial and $f(X) \in \mathbf{A}_{v}[[X]]$, then the operation

$$
P(D) f(X): \mathbf{A}_{v}[[X]] \rightarrow \mathbf{A}_{v}[[X]]
$$

is well defined. Indeed, because $P(Z)$ is a divided polynomial, no convergence problems arise. The following lemma, whose proof is elementary, was pointed out to me by W. Sinnott:

Lemma 3.17. Let " $X$ " denote the operator "multiplication by $X$ " on power series. Let $\left[\frac{D^{k}}{k !}, X\right]$ denote the commutator of $\frac{D^{k}}{k !}$ and $X$. Then

$$
\left[\frac{D^{k}}{k !}, X\right]=\frac{D^{(k-1)}}{(k-1) !} \text {. }
$$

Definition 3.18. Let $a \in \mathbf{A}$ and $\mu=P_{\mu}=\sum a_{k} X^{k} \in M_{v}\left(\mathbf{Z}_{p}\right)$. Then, in the notation of Proposition 3.8, we set $D(a)=P_{a}(D) \in \mathbf{A}_{v}\{\{D\}\}$, and

$$
D(a)(\mu)=P_{a}(D) P_{\mu}(X) \in M_{v}\left(\mathbf{Z}_{p}\right)
$$

Note that the above definition makes sense since we have seen (Proposition 3.8) that for $a \in \mathbf{A}, P_{a}(Z)$ is a divided polynomial.

Proposition 3.19. Let $a, b \in \mathbf{A}$. Then

$$
D(a+b) \mu=D(a) D(b) \mu .
$$


Proof. We have seen that $P_{(a+b)}(Z)=P_{a}(Z) P_{b}(Z)$. The result follows directly.

Thus we have defined an action of $\mathbf{A}$ on the $\mathbf{A}_{v}$-valued measures on $\mathbf{Z}_{p}$. Next we discuss what can be done at the prime $\infty$ of $\mathbf{k}$.

Let $\overline{\mathbf{K}}$ be a fixed algebraic closure of $\mathbf{K}$. We equip $\overline{\mathbf{K}}$ with the canonical extension of the normalized absolute value, $\mid$ ? $\left.\right|_{\infty}$, on $\mathbf{K}$. Then, using the $v$-adic theory as a guide we make the following definition:

Definition 3.20. Let $\alpha \in \overline{\mathbf{K}}$. Then we set

$$
D(\alpha)=\sum_{k \geq 0} \frac{G_{k}(\alpha)}{\Pi(k)} \frac{D^{k}}{k !} .
$$

The $v$-adic theory then assures us that $D(\alpha+\beta)=D(\alpha) D(\beta)$. Indeed the formalism is exactly the same.

Lemma 3.21. Let $\alpha \in \overline{\mathbf{K}}$. Then as $k \rightarrow \infty, \frac{G_{k}(\alpha)}{\Pi(k)} \rightarrow 0$ in $\overline{\mathbf{K}}$.

Proof. This is an easy exercise with nonarchimedean estimates.

Thus, the above lemma assures us that the obvious $\infty$-adic version of definition 3.18 gives us an action of $\mathbf{K}$ on $B M_{\infty}\left(\mathbf{Z}_{P}\right)$. A very important problem now arises: give a definition of these actions that does not depend on the explicit realization of these elements as divided power series, etc.

Finally, there is a remarkable, and as yet not fully understood, connection between gamma functions and the actions just given which we now present. The deeper understanding of this connection also appears as a crucial problem.

Definition 3.22. Let $y \in \mathbf{Z}_{p}$ be written as

$$
y=\sum_{t=0}^{\infty} \alpha_{t} r^{t}, \quad 0 \leq \alpha_{t}<r
$$

Let $x$ be a variable. Then we set

$$
\begin{gathered}
g(x, y)=\prod_{t=0}^{\infty}\left(1+\frac{e_{t}(x)}{D_{t}}\right)^{\alpha_{t}}, \\
\Pi_{0}(x, y)=g(x, y)^{-1},
\end{gathered}
$$

and

$$
\Gamma_{0}(x, y)=\Pi_{0}(x, y-1) .
$$

The function $\Gamma_{0}$ has roughly the same relationship to the exponential of the Carlitz module as Euler's $\Gamma$ has to the function $\sin (x)$. The subscript " 0 " is used to signify that in order to obtain the "proper" definition of a gamma function, the function $\Gamma_{0}$ must be modified. One multiplies it by the function obtained by interpolating the Carlitz factorials $\Pi(k)$ at the prime $\infty$ (see [6]).

In [6] the following result is established. It tells us that the Carlitz functions are precisely the Mahler coefficients of the function $g(x, y)$. 
Theorem 3.23. We have

$$
g(x, y)=\sum_{k \geq 0}\left(\begin{array}{l}
y \\
k
\end{array}\right) \frac{G_{k}(x)}{\Pi(k)} .
$$

Note that if $a \in \mathbf{A}$ then so does $g(a, y)$ for any $y$. Indeed, the above expansion clearly terminates.

The reader will immediately see the similarity between the above expansion and the definition of the operators $D(\alpha)$. This leads directly to our next result:

Theorem 3.24. Let $y \in \mathbf{Z}_{p}$.

(a) (v-adic version) For $a \in \mathbf{A}$ we have

$$
\int_{\mathbf{Z}_{p}} d D(a)\left(\delta_{y}\right)(x)=g(a, y) .
$$

(b) ( $\infty$-adic version) For $\alpha \in \mathbf{K}$ we have

$$
\int_{\mathbf{Z}_{p}} d D(\alpha)\left(\delta_{y}\right)(x)=g(\alpha, y) .
$$

Proof. Let $f(X)$ be a power series representing some measure $\mu$ on $\mathbf{Z}_{p}$. By definition, $f(0)$ is the integral of $\mu$ over all of $\mathbf{Z}_{p}$.

Now we have seen that $\delta_{y}$ corresponds to the power series $(1+X)^{y}$. Applying $D(\alpha)$ to it, we obtain

$$
\sum_{k \geq 0}\left(\begin{array}{l}
y \\
k
\end{array}\right) \frac{G_{k}(x)}{\Pi(k)}(1+X)^{y-k} .
$$

Part (b) now follows immediately from the above remark and Theorem 3.23. Part (a) follows in a completely similar fashion.

Let $\mathbf{e}_{C}(x)$ be the exponential of the Carlitz module [2], with period $\xi$. Let $\mathbf{e}(x)$ be the exponential of $\mathbf{A}$ viewed as a rank one lattice; so

$$
\frac{\mathbf{e}_{C}(\xi x)}{\xi}=\mathbf{e}(x) \text {. }
$$

Let $G=\mathbf{F}_{r}^{*} \times \pm 1$. We define an action $\rho$ of $G$ on $\overline{\mathbf{K}} \times \mathbf{Z}_{p}$ by

$$
(x, y)^{\rho(\zeta, 1)}=(\zeta x, y),
$$

and

$$
(x, y)^{\rho(\zeta,-1)}=(\zeta x, 1-y)
$$

Put $s=(x, y)$. Then one sees easily the following functional equation for $g(x, y)$.

\section{Proposition 3.25.}

$$
\prod_{\sigma \in G} g\left(s^{\rho(\sigma)}\right)=\left(\frac{\mathbf{e}(x)}{x}\right)^{(r-1)}
$$


The second part of Theorem 3.24 is now equivalent to

Theorem 3.26. Let $\alpha \in \mathbf{K}$. Put $s=(\alpha, y)$. Then

$$
\int_{\mathbf{z}_{p}} d D(\alpha)\left(\delta_{y}\right)(x)=\left(\frac{\mathbf{e}(\alpha)}{\alpha}\right)^{(r-1)} \prod_{\sigma \neq 1} \Pi_{0}\left(s^{\rho(\sigma)}\right) .
$$

The functions $g(x, y), \Pi_{0}(x, y)$, etc., are defined on the two-variable space $\overline{\mathbf{K}} \times \mathbf{Z}_{p}$ (and, as mentioned in our next section, $L$-series are defined naturally on the space $\mathbf{S}_{\infty}=\overline{\mathbf{K}}^{*} \times \mathbf{Z}_{p}$ ). It may thus turn out that integration over $\mathbf{Z}_{p}$ will ultimately play the role that is classically played by integration over $i \mathbf{R} \subset \mathbf{C}$.

3.3. The formal Mellin transform of $\zeta(s)$. In this last section we follow the classical approach of our first section to obtain "formal Mellin transforms" in the arithmetic of function fields. However, whereas classically one obtains the power series $\frac{T}{1-T}$, the $v$-adic theory leads us to expect divided power series for function fields.

Definition 3.27. Let $0<c<r$, and $j$ be a positive integer. We put $i=i(c, j)$ $=c r^{j}+\left(r^{j}-1\right)$. We call numbers of the form $i(c, j)$ and 0 magic numbers.

The magic numbers are of importance because they appear in the KummerVandiver conjecture for function fields, (see [5]).

In the arithmetic of function fields one has the analog of the Riemann zeta function defined by

$$
\zeta(s)=\sum_{\substack{n \in \mathbf{A} \\ n \text { monic }}} n^{-s} .
$$

This sum has an obvious interpretation as a function from the positive integers into $\mathbf{K}$, but can actually be analytically continued to the space $\mathbf{S}_{\infty}=\overline{\mathbf{K}}^{*} \times \mathbf{Z}_{p}$. This is accomplished by "summing according to degree" $[2,3]$. One remarkable fact about $\zeta(s)$ on $\mathbf{S}_{\infty}$ is its "special polynomials" which are obtained as special values at negative integers and which are presented in the next definition.

Definition 3.28. Let $x$ be an indeterminate and let $i$ be a nonnegative integer. We define $z(x,-i)$ by

$$
z(x,-i)=\sum_{j=0}^{\infty} x^{-j}\left(\sum_{n \text { monic of degree } j} n^{i}\right) .
$$

Although $z(x,-i)$ is a priori defined as an infinite series, it is, in fact, a polynomial in $x^{-1}$ with $\mathbf{A}$ coefficients. Thus, for instance, the value of $z(x,-i)$ at $x=1$ is the value of $\zeta(s)$ at $s=-i$, etc.

Let $v$ be a prime of $\mathbf{A}$. The polynomials $z(x,-i)$ appear as the moments of the $v$-adic measure $\mu_{x}$ on $\mathbf{A}_{v}$ defined by

$$
\mu_{x}=\sum_{n \text { monic }} x^{-\operatorname{deg}(n)} \delta_{n}
$$

Let $U \subseteq \mathbf{A}_{v}$ be a compact open subset. By summing according to degree, one sees that $\mu_{x}(U)$ is also a polynomial in $x^{-1}$ with A-coefficients. 
Definition 3.29. The formal Mellin transform, $M_{\zeta}(Z, x)$, of $\zeta(s)$ is defined to be the divided power series given by

$$
M_{\zeta}(Z, x)=\sum_{j=0}^{\infty}\left(\sum_{n \text { monic of degree } j} P_{n}(Z)\right) x^{-j} .
$$

Since a measure is determined by its moments, and since $z(x,-i)$ is a polynomial, it follows that $M_{\zeta}(Z, x)$ can be written as

$$
M_{\zeta}(Z, x)=\sum_{i \geq 0} m_{\zeta}(x,-i) \frac{Z^{i}}{i !},
$$

where $m_{\zeta}(x,-i)$ is a polynomial in $x^{-1}$.

By using moments, it is possible to calculate $m_{\zeta}(x,-i)$. This was done originally by Dinesh Thakur by hand computations under the assumption that $x=1$ (so we are in the case of "special-values"). Thakur's computations, and partial results, suggested a connection with magic numbers. Following up on this, the author put the full two-variable calculation on a machine. It was found experimentally that

1. $m_{\zeta}(x, 0)=1$.

2. $m_{\zeta}(x,-i), i>0$, is nonvanishing if and only if $i$ is a magic number.

3. Let $i=i(c, j)$. Then we have
(a) If $c<r-1$, then $m_{\zeta}(x,-i)=(-1)^{j} x^{-j}$.
(b) If $c=r-1$, then $m_{\zeta}(x,-i)=(-1)^{j}\left(1-x^{-1}\right) x^{-j}$.

The results implied by the above computations have recently been proved by Dinesh Thakur [10]. Thakur's work establishes once again the central role that magic numbers appear to play in the theory.

Note the remarkable simplicity of the above formula for $m_{\zeta}(x,-i)$. Among other things, the coefficients of $m_{\zeta}(x,-i)$, which are a priori in $\mathbf{k}$, turn out to be elements of $\mathbf{F}_{p}$.

3.3.1. Variants. Let $\mathbf{L}$ be a finite extension of $\mathbf{k}$ and let $O_{\mathbf{L}}$ be its ring of A-integers. Let $\rho$ be an ideal of $O_{\mathrm{L}}$ and $N \rho$ the monic generator of its norm. Associated to $O_{\mathrm{L}}$ there is the analog of its Dedekind zeta function, $\zeta_{O_{\mathrm{L}}}(s)$, which is defined in the obvious fashion (see [3]). The formal Mellin transform of $\zeta_{O_{\mathrm{L}}}(s)$ is then defined to be

$$
M_{\zeta_{\alpha_{\mathrm{L}}}}(Z, x)=\sum_{\rho} P_{N \rho}(Z) x^{-\operatorname{deg}(N \rho)} .
$$

As before, we sum according to degree, and we write

$$
M_{\zeta_{\sigma_{\mathrm{L}}}}(Z, x)=\sum_{i \geq 0} m_{\zeta_{o_{\mathrm{L}}}}(x,-i) \frac{Z^{i}}{i !} .
$$

Proposition 3.30. The functions $m_{\zeta_{o_{\mathrm{L}}}}(x,-i)$ are polynomials in $x^{-1}$ with $\mathbf{A}$ coefficients. 
Proof (Sketch). Let $v$ be a prime of $\mathbf{A}$ and let $\mathbf{L}\left(v^{\infty}\right)$ be the field obtained by adjoining to $\mathbf{L}$ all $v$-power torsion points of the Carlitz module. Clearly $G=\operatorname{Gal}\left(\mathbf{L}\left(v^{\infty}\right) / \mathbf{L}\right)$ is a subgroup of $\mathbf{A}_{v}^{*}$. The results of [3] allow us to construct a measure $\mu_{x}^{1}$ on $G$ whose moments (under the norm map to $\mathbf{A}_{v}^{*}$ ) are precisely the special polynomials of $\zeta_{O_{\mathrm{L}}}(s)$ except that the $v$-adic Euler factors are removed.

The measure $\mu_{x}^{1}$ can be pushed forward to obtain a measure on $\mathbf{A}_{v}^{*}$. Moreover, the techniques of [4, Theorem 2.9] can be used to extend $\mu_{x}^{1}$ to a measure $\mu_{x}$ on $\mathbf{A}_{v}$ whose moments are precisely the special polynomials. Since a measure is determined by its moments, $\mu_{x}$ has to correspond to the formal Mellin transform of the Dedekind zeta function of $O_{\mathrm{L}}$ by analytic continuation (since, for $x$ sufficiently large $v$-adically, the formal Mellin transform clearly gives the right measure).

Thus, we see that the formal Mellin transform gives the correct measure at all the primes of $\mathbf{A}$. The result now follows directly.

It would be very interesting to compute these divided power series. If it actually turns out that their coefficients also are polynomials with $\mathbf{F}_{p}$-coefficients, then it makes sense to inquire if these formal Mellin transforms are somehow obtained from characteristic 0 in the same way that the measures on $\mathbf{A}_{v}^{*}$ are (see $[2,3])$; i.e., through the use of Stickelberger elements.

Historical Note. The class of integers which we call "magic numbers" originally occurred in computations of the degrees of the elements $\beta(i)$ of [2] by P. Emery Thomas. It turns out that the formula found by Thomas considerably simplifies for magic numbers.

\section{REFERENCES}

1. E.-U. Gekeler, On power sums of polynomials over finite fields, J. Number Theory 30 (1988), 11-27.

2. D. Goss, The arithmetic of function fields 2: The 'cyclotomic' theory, J. Algebra 81 (1983), 107-149.

3. _ The theory of totally-real function fields, Applications of Algebraic $K$-Theory to Algebraic Geometry, Vol. 55, part II, Amer. Math. Soc., Providence, R. I., 1986, pp. 449-477.

4. D. Goss and W. Sinnott, Special values of Artin L-series, Math. Ann. 275 (1986), 529-537.

5. D. Goss, The $\Gamma$-function in the arithmetic of function fields, Duke Math. J. 56 (1988), 163-191.

6. __ Fourier series, measures and divided power series in the theory of function fields, $K$ Theory 1 (1989), 533-555.

7. _ Harmonic analysis and the flow of a Drinfeld module, J. Algebra (to appear).

8. N. Katz, P-adic L-functions via moduli of elliptic curves, Proc. Sympos. Pure Math., vol. 29, Amer. Math. Soc., Providence, R. I., 1975, pp. 479-506.

9. S. Lang, Cyclotomic fields, Springer, 1978.

10. D. Thakur, Zeta measure associated to $\mathbf{F}_{q}[T]$, J. Number Theory 35 (1990) 1-17.

Department of Mathematics, Ohio State University, Columbus, Ohio 43210-1174

E-mail address: goss@coltrane.mps.ohio-state.edu 